\title{
Simplicity and Commutative Bases of Derivations in Polynomial and Power Series Rings
}

\author{
Rene Baltazar \\ Universidade Federal do Rio Grande do Sul (UFRGS), Campus do Vale, Código Postal 15051, 91501-970 Porto Alegre, RS, Brazil \\ Correspondence should be addressed to Rene Baltazar; rene.baltazar@ufrgs.br
}

Received 8 October 2013; Accepted 24 November 2013

Academic Editors: H. Chen and A. Zimmermann

Copyright (C) 2013 Rene Baltazar. This is an open access article distributed under the Creative Commons Attribution License, which permits unrestricted use, distribution, and reproduction in any medium, provided the original work is properly cited.

\begin{abstract}
The first part of the paper will describe a recent result of Retert in (2006) for $k\left[x_{1}, \ldots, x_{n}\right]$ and $k\left[\left[x_{1}, \ldots, x_{n}\right]\right]$. This result states that if $\mathfrak{D}$ is a set of commute $k$-derivations of $k[x, y]$ such that both $\partial_{x} \in \mathfrak{D}$ and the ring is $\mathfrak{D}$-simple, then there is $d \in \mathfrak{D}$ such that $k[x, y]$ is $\left\{\partial_{x}, d\right\}$-simple. As for applications, we obtain relationships with known results of A. Nowicki on commutative bases of derivations.
\end{abstract}

\section{Introduction}

Let $k$ be a field of characteristic zero and $R$ denote either the ring $k\left[x_{1}, \ldots, x_{n}\right]$ of polynomials over $k$ or the ring $k\left[\left[x_{1}, \ldots, x_{n}\right]\right]$ of formal power series over $k$.

A $k$-derivation $d: R \rightarrow R$ of $R$ is a $k$-linear map such that $d(a b)=d(a) b+a d(b)$ for any $a, b \in R$. Denoting by $\operatorname{Der}_{k}(R)$ the set of all $k$-derivations of $R$, let $\mathfrak{D} \subset \operatorname{Der}_{k}(R)$ be a nonempty family of $k$-derivations. An ideal $I$ of $R$ is called $\mathfrak{D}$-stable if $d(I) \subset I$ for all $d \in \mathfrak{D}$. For example, the ideals 0 and $R$ are always $\mathfrak{D}$-stable. If $R$ has no other $\mathfrak{D}$-stable ideal it is called $\mathfrak{D}$-simple. When $\mathfrak{D}=\{d\}, d$ is often called a simple derivation.

The commuting derivations have been studied by several authors: Li and $\mathrm{Du}$ [1], Maubach [2], Nowicki [3], Petravchuk [4], Retert [5], Van den Essen [6]. For example, it is well known that each pair of commuting linear operators on a finite dimensional vector space over an algebraically closed field has a common eigenvector; in [4], Petravchuk proved an analogous statement for derivation of $k[x, y]$ over any field $k$ of characteristic zero. More explicitly, if two derivations of $k[x, y]$ are linearly independent over $k$ and commute, then they have a common Darboux polynomial or they are Jacobian derivations; in $[1]$, the authors proved the same result for $k\left[x_{1}, \ldots, x_{n}\right]$ and $k\left[\left[x_{1}, \ldots, x_{n}\right]\right]$. However, we observe that this result has already been proved by Nowicki in (Now86) for both rings. Another interesting result was proved by Nowicki in [3, Theorem 5] which says that the famous Jacobian conjecture in $k\left[x_{1}, \ldots, x_{n}\right]$ is equivalent to the assertion that every commutative basis of $\operatorname{Der}_{k}(R)$ is locally nilpotent.

Let $\mathfrak{D}$ be a set of commute $k$-derivations of $k[x]$; then $k[x]$ is $\mathfrak{D}$-simple if and only if it is $d$-simple for some $k$-derivation $d \in \mathfrak{D}$ (see [5, Corollary 2.10]). In $k[x, y]$, as pointed out in [5], up to scalar multiples, these are only sets $\mathfrak{D}$ of two commuting, nonsimple $k$-derivations such that both $\partial_{x} \in \mathfrak{D}$ and $R$ is $\mathfrak{D}$-simple. Motivated by this, we analyze this result in [5] for $R$ and then we propose some connections with known results on commutative bases of derivations in $R$. More precisely, the derivations $\partial_{x_{1}}, \ldots, \partial_{x_{n-1}}$ are not simple $k$-derivations of $R$; however, as will be shown, they can be part of a set $\mathfrak{D}$ of $n$ commuting, nonsimple $k$-derivations such that $R$ is Do-simple. A trivial example is $\mathfrak{D}=\left\{\partial_{x_{1}}, \ldots, \partial_{x_{n}}\right\}$. Using the notations in [3], we give a nontrivial commutative base containing only nonsimple $k$-derivations of the free $R$-module $\operatorname{Der}_{k}(R)$ such that $R$ is $\mathfrak{D}$-simple and if the Jacobian conjecture is true in $k\left[x_{1}, \ldots, x_{n}\right]$, as a consequence of [3, Theorem 5], we obtain a family of locally nilpotent derivations. 


\section{Commuting Derivations and Simplicity}

Lemma 1. The set of all $k$-derivations of $R$ that commute with $\partial_{x_{1}}, \ldots, \partial_{x_{n-1}}$ is

$$
\left\{p\left(x_{n}\right) \partial_{x_{1}}+q_{1}\left(x_{n}\right) \partial_{x_{2}}+\cdots+q_{n-1}\left(x_{n}\right) \partial_{x_{n}}\right\}
$$

such that $p, q_{1}, \ldots, q_{n-1} \in k\left[x_{n}\right]$ (or $k\left[\left[x_{n}\right]\right]$ if $R=k\left[\left[x_{1}, \ldots\right.\right.$, $\left.\left.x_{n}\right]\right]$ ).

Proof. It is clear that all derivations of this form commute with the derivations $\partial_{x_{1}}, \ldots, \partial_{x_{n-1}}$. For the converse, let

$$
\begin{aligned}
d^{*}= & p^{*}\left(x_{1}, \ldots, x_{n}\right) \partial_{x_{1}}+q_{1}^{*}\left(x_{1}, \ldots, x_{n}\right) \partial_{x_{2}} \\
& +\cdots+q_{n-1}^{*}\left(x_{1}, \ldots, x_{n}\right) \partial_{x_{n}}
\end{aligned}
$$

be a $k$-derivation of $R$ that commutes with $\partial_{x_{1}}, \ldots, \partial_{x_{n-1}}$. Then

$$
0=d^{*}\left(\partial_{x_{i}}\left(x_{1}\right)\right)=\partial_{x_{i}}\left(d^{*}\left(x_{1}\right)\right)=\partial_{x_{i}}\left(p^{*}\left(x_{1}, \ldots, x_{n}\right)\right)
$$

for all $i=1, \ldots, n-1$. Thus, $p^{*}\left(x_{1}, \ldots, x_{n}\right) \in k\left[x_{n}\right]\left(k\left[\left[x_{n}\right]\right]\right)$. Similarly, we can prove that $q_{i}^{*}\left(x_{1}, \ldots, x_{n}\right) \in k\left[x_{n}\right]\left(k\left[\left[x_{n}\right]\right]\right)$.

Let $\mathfrak{D}=\left\{\delta_{1}, \ldots, \delta_{s}\right\}$ be any finite set of $k$-derivation of $R$ that commutes with $\partial_{x_{1}}, \ldots, \partial_{x_{n-1}}$ but not necessarily with each other. By Lemma 1, each $\delta_{i}$ is of the form

$$
\delta_{i}=p_{i}\left(x_{n}\right) \partial_{x_{1}}+q_{(1, i)}\left(x_{n}\right) \partial_{x_{2}}+\cdots+q_{(n-1, i)}\left(x_{n}\right) \partial_{x_{n}} .
$$

We denote by $v_{n-1}\left(x_{n}\right)$ the greatest common divisor of $q_{(n-1,1)}, \ldots, q_{(n-1, s)}$. We have the following characterization for the simplicity of $R$.

Lemma 2. Using the notations above, $R$ is $\mathfrak{D}$-simple if and only if $v_{n-1}\left(x_{n}\right)$ is a unit in $k\left[x_{n}\right]$ (or $\left.k\left[\left[x_{n}\right]\right]\right)$.

Proof. If all $q_{(n-1, i)}=0$, so all the $k$-derivations in $\mathfrak{D}$ stabilize the nonzero ideal $\left\langle x_{n}\right\rangle$; in this case, $R$ is not $\mathfrak{D}$-simple. Then we assume that some $q_{(n-1, i)} \neq 0$. If $v_{n-1}\left(x_{n}\right)$ is not a unit, each $\delta_{i}$ stabilizes the nontrivial ideal $\left\langle v_{n-1}\left(x_{n}\right)\right\rangle$. Therefore $R$ is not D-simple.

Conversely, assume that $v_{n-1}\left(x_{n}\right)$ is a unit and notice that, in this case, there are polynomials $r_{i}\left(x_{n}\right)$ such that

$$
\sum_{i=1}^{s} r_{i}\left(x_{n}\right) q_{(n-1, i)}\left(x_{n}\right)=v_{n-1}\left(x_{n}\right)
$$

multiplicand by the inverse of $v_{n-1}\left(x_{n}\right)$, we may assume that $\sum_{i=1}^{s} r_{i}\left(x_{n}\right) q_{(n-1, i)}\left(x_{n}\right)=1$. Without loss of generality, let $\delta_{1}=$ $\partial_{x_{1}}, \ldots, \delta_{n-1}=\partial_{x_{n-1}}$; thus,

$$
\sum_{i=n}^{s} r_{i}\left(x_{n}\right) q_{(n-1, i)}\left(x_{n}\right)=1
$$

Let $I$ be a $\mathfrak{D}$-ideal. Then since $I$ is stabilized by each $\delta_{i}, I$ is stabilized by $r_{i}\left(x_{n}\right) \delta_{i}$ and, then, by the $k$-derivation

$$
\begin{aligned}
\sum_{i=n}^{s} r_{i}\left(x_{n}\right) \delta_{i}= & \left(\sum_{i=n}^{s} r_{i}\left(x_{n}\right) p_{i}\left(x_{n}\right)\right) \partial_{x_{1}} \\
& +\cdots+\left(\sum_{i=n}^{s} r_{i}\left(x_{n}\right) q_{(n-1, i)}\left(x_{n}\right)\right) \partial_{x_{n}} .
\end{aligned}
$$

Therefore, $I$ is stabilized by $\partial_{x_{1}}, \ldots, \partial_{x_{n-1}}$ and a $k$-derivation of the form

$$
u_{1}\left(x_{n}\right) \partial_{x_{1}}+\cdots+u_{n-1}\left(x_{n}\right) \partial_{x_{n-1}}+\partial_{x_{n}}
$$

for $u_{i}\left(x_{n}\right) \in k\left[x_{n}\right]\left(k\left[\left[x_{n}\right]\right]\right)$. Thus $I$ is stabilized by $\partial_{x_{1}}, \ldots, \partial_{x_{n-1}}, \partial_{x_{n}}$ and, then, we deduce that $I$ must be a trivial ideal.

Note that until now we only assume that all the $k$ derivations commute with $\partial_{x_{1}}, \ldots, \partial_{x_{n-1}}$ not that all elements commute with each other. Using the previous lemmas, the following theorem will show that if $R$ is $\mathfrak{D}$-simple under a set $\mathfrak{D}$ of commuting $k$-derivations that contains $\partial_{x_{1}}, \ldots, \partial_{x_{n-1}}$, then $R$ is simple under a subset of $n$ commuting nonsimple $k$ derivations.

Theorem 3. Let $\mathfrak{D}$ be a set of $k$-derivations of $R$ such that $\partial_{x_{1}}, \ldots, \partial_{x_{n-1}} \in \mathfrak{D}$. Then the derivations of $\mathfrak{D}$ commute with each other if and only if one of the following two cases holds.

(a) Each element $\delta_{i}$ of $\mathfrak{D}$ has the form $\delta_{i}=h_{1}^{i}\left(x_{n}\right) \partial_{x_{1}}+\cdots+$ $h_{n-1}^{i}\left(x_{n}\right) \partial_{x_{n-1}}$, for some $h_{j}^{i}\left(x_{n}\right) \in k\left[x_{n}\right]$ (or $\left.k\left[\left[x_{n}\right]\right]\right)$.

(b) There exists $v_{1}\left(x_{n}\right), \ldots, v_{n}\left(x_{n}\right) \in k\left[x_{n}\right]$ (or $k\left[\left[x_{n}\right]\right]$ ), such that for each $\delta_{i} \in \mathfrak{D}$ there are scalars $\lambda_{i}, c_{1}^{i}, \ldots, c_{n-1}^{i} \in k$ such that

$$
\delta_{i}=\sum_{l=1}^{n-1}\left(\lambda_{i} v_{l}\left(x_{n}\right)+c_{l}^{i}\right) \partial_{x_{l}}+\lambda_{i} v_{n}\left(x_{n}\right) \partial_{x_{n}} .
$$

If, in addition, $R$ is $\mathfrak{D}$-simple, then $v_{n}\left(x_{n}\right)$ must be some nonzero scalar $\beta$ and, also, some $\lambda_{j}$ is not zero. In this case, $R$ is also simple under the subset

$$
\left\{\partial_{x_{1}}, \ldots, \partial_{x_{n-1}}, \sum_{l=1}^{n-1}\left(\lambda_{j} v_{l}\left(x_{n}\right)+c_{l}^{j}\right) \partial_{x_{l}}+\lambda_{j} \beta \partial_{x_{n}}\right\} .
$$

Proof. If either of the conditions is met, it is clear that all $k$ derivations of $\mathfrak{D}$ will commute.

Conversely, let $\mathfrak{D}=\left\{\delta_{1}, \ldots, \delta_{m}\right\}$ be according to the hypotheses of the theorem. By Lemma 1 , each $\delta_{i}$ is of the form $\delta_{i}=p_{i}\left(x_{n}\right) \partial_{x_{1}}+q_{(1, i)}\left(x_{n}\right) \partial_{x_{2}}+\cdots+q_{(n-1, i)}\left(x_{n}\right) \partial_{x_{n}}$.

If all $q_{(n-1, i)}\left(x_{n}\right)$ are zero, then the first case holds. So, without loss of generality, we assume that $q_{(n-1,1)}\left(x_{n}\right) \neq 0$ and observe that

$$
\begin{aligned}
\delta_{i}\left(\delta_{1}\left(x_{n}\right)\right) & =\delta_{i}\left(q_{(n-1,1)}\left(x_{n}\right)\right) \\
& =q_{(n-1, i)}\left(x_{n}\right) \partial_{x_{n}}\left(q_{(n-1,1)}\left(x_{n}\right)\right), \\
\delta_{1}\left(\delta_{i}\left(x_{n}\right)\right) & =\delta_{1}\left(q_{(n-1, i)}\left(x_{n}\right)\right) \\
& =q_{(n-1,1)}\left(x_{n}\right) \partial_{x_{n}}\left(q_{(n-1, i)}\left(x_{n}\right)\right) .
\end{aligned}
$$


Since $\delta_{i}$ and $\delta_{1}$ commute,

$$
\begin{aligned}
& q_{(n-1, i)}\left(x_{n}\right) \partial_{x_{n}}\left(q_{(n-1,1)}\left(x_{n}\right)\right) \\
& =q_{(n-1,1)}\left(x_{n}\right) \partial_{x_{n}}\left(q_{(n-1, i)}\left(x_{n}\right)\right) .
\end{aligned}
$$

Then, this equation must also hold in the ring of fractions; hence we deduce that

$$
\frac{q_{(n-1, i)}\left(x_{n}\right) \partial_{x_{n}}\left(q_{(n-1,1)}\left(x_{n}\right)\right)-q_{(n-1,1)}\left(x_{n}\right) \partial_{x_{n}}\left(q_{(n-1, i)}\left(x_{n}\right)\right)}{\left(q_{(n-1,1)}\left(x_{n}\right)\right)^{2}}
$$$$
=0 .
$$

In other words,

$$
\left(\frac{q_{(n-1, i)}\left(x_{n}\right)}{q_{(n-1,1)}\left(x_{n}\right)}\right)^{\prime}=0 .
$$

Then there is some $\lambda_{i} \in k$ such that $q_{(n-1, i)}\left(x_{n}\right)=$ $\lambda_{i} q_{(n-1,1)}\left(x_{n}\right)$.

Now, we observe that

$$
\begin{aligned}
\delta_{i}\left(\delta_{1}\left(x_{1}\right)\right) & =\delta_{i}\left(p_{1}\left(x_{n}\right)\right) \\
& =q_{(n-1, i)}\left(x_{n}\right) \partial_{x_{n}}\left(p_{1}\left(x_{n}\right)\right), \\
\delta_{1}\left(\delta_{i}\left(x_{1}\right)\right) & =\delta_{1}\left(p_{i}\left(x_{n}\right)\right) \\
& =q_{(n-1,1)}\left(x_{n}\right) \partial_{x_{n}}\left(p_{i}\left(x_{n}\right)\right) .
\end{aligned}
$$

Since $\delta_{i}$ and $\delta_{1}$ commute and both $k\left[x_{n}\right]$ and $k\left[\left[x_{n}\right]\right]$ are domains,

$$
\lambda_{i} \partial_{x_{n}}\left(p_{1}\left(x_{n}\right)\right)=\partial_{x_{n}}\left(p_{i}\left(x_{n}\right)\right)
$$

Then there is some $c_{1}^{i} \in k$ such that $p_{i}\left(x_{n}\right)=\lambda_{i} p_{1}\left(x_{n}\right)+c_{1}^{i}$. Finally, making the same argument for the other variables, we prove the desired result.

Now we suppose, in addition, that $R$ is $\mathfrak{D}$-simple. By Lemma 2, the greatest common divisor of $q_{(n-1,1)}\left(x_{n}\right), \ldots, q_{(n-1, m)}\left(x_{n}\right)$ must be a unit. However, we have demonstrated that all the $q_{(n-1, i)}\left(x_{n}\right)$ are scalar multiples; then at least one of the $q_{(n-1, i)}\left(x_{n}\right)$ must be a unit; we assume that $q_{(n-1, j)}\left(x_{n}\right)$ is a unit. Since $I$ is stabilized by

$$
\begin{aligned}
& \left\{\partial_{x_{1}}, \ldots, \partial_{x_{n-1}}, p_{j}\left(x_{n}\right) \partial_{x_{1}}+q_{(1, j)}\left(x_{n}\right) \partial_{x_{2}}\right. \\
& \left.+\cdots+q_{(n-1, j)}\left(x_{n}\right) \partial_{x_{n}}\right\}
\end{aligned}
$$

$I$ must be trivial because, in this case, $I$ is stabilized by $\partial_{x_{1}}, \ldots, \partial_{x_{n-1}}, \partial_{x_{n}}$. Therefore, $R$ is $\left\{\partial_{x_{1}}, \ldots, \partial_{x_{n-1}}, p_{j}\left(x_{n}\right) \partial_{x_{1}}+\right.$ $\left.q_{(1, j)}\left(x_{n}\right) \partial_{x_{2}}+\cdots+q_{(n-1, j)}\left(x_{n}\right) \partial_{x_{n}}\right\}$-simple which completes the proof.

Remark 4. Nowicki in [7, Theorem 2.5.5] proved that every $k$-derivation of a commutative base of $\operatorname{Der}_{k}\left(k\left[x_{1}, \ldots, x_{n}\right]\right)$ is a special $k$-derivation. This means that the divergence $d^{*}$ of $d$ is 0 . Moreover, it is easy to prove that the set

$$
\left\{\partial_{x_{1}}, \ldots, \partial_{x_{n-1}}, \sum_{l=1}^{n-1}\left(\lambda_{j} v_{l}\left(x_{n}\right)+c_{l}^{j}\right) \partial_{x_{l}}+\lambda_{j} \beta \partial_{x_{n}}\right\}
$$

obtained by the previous theorem is a commutative base of $\operatorname{Der}_{k}\left(k\left[x_{1}, \ldots, x_{n}\right]\right)$. Thus, in particular, $\sum_{l=1}^{n-1}\left(\lambda_{j} v_{l}\left(x_{n}\right)+\right.$ $\left.c_{l}^{j}\right) \partial_{x_{l}}+\lambda_{j} \beta \partial_{x_{n}}$ is a special derivation. However, this is easily verified since

$$
d^{*}=\sum_{l=1}^{n-1} \partial_{x_{l}}\left(\lambda_{j} v_{l}\left(x_{n}\right)+c_{l}^{j}\right)+\partial_{x_{n}}\left(\lambda_{j} \beta\right)=0
$$

Corollary 5. Let $\mathfrak{D}$ be a set of commute $k$-derivations of $R$ such that $R$ is $\mathfrak{D}$-simple and $\partial_{x_{1}}, \ldots, \partial_{x_{n-1}} \in \mathfrak{D}$. Then, there exists $d \in \mathfrak{D}$ and there exist elements $f_{1}, \ldots, f_{n} \in R$ such that $d\left(f_{n}\right)=1$ and $d\left(f_{i}\right)=0$, for any $i=1, \ldots, n-1$, so that $R$ is $\left\{\partial_{x_{1}}, \ldots, \partial_{x_{n-1}}, d\right\}$-simple.

Proof. By the previous theorem, we know that there is $d \in \mathfrak{D}$ of the form

$$
d=\sum_{l=1}^{n-1}\left(\lambda_{j} v_{l}\left(x_{n}\right)+c_{l}^{j}\right) \partial_{x_{l}}+\lambda_{j} \beta \partial_{x_{n}}
$$

such that $R$ is $\left\{\partial_{x_{1}}, \ldots, \partial_{x_{n-1}}, d\right\}$-simple. Since $\beta$ and $\lambda_{j}$, in the theorem, are nonzero scalars, we denote $f_{n}=\left(\lambda_{j} \beta\right)^{-1} x_{n}$; thus $d\left(f_{n}\right)=1$.

Let $f_{l}^{*} \in k\left[x_{n}\right]\left(\right.$ or $\left.k\left[\left[x_{n}\right]\right]\right)$ such that $d\left(f_{l}^{*}\right)=\lambda_{j} v_{l}\left(x_{n}\right)+$ $c_{l}^{j}$. Since $c(k)=0, f_{l}^{*}$ exist. Then, let $f_{l}=x_{l}-f_{l}^{*}$; hence $d\left(f_{l}\right)=0$, for any $l=1, \ldots, n-1$. This completes the proof.

Remark 6. The previous corollary is a particular case of an important theorem about the characterization of commutative basis of $\operatorname{Der}_{k}(R)$ However, in our case the proof is more evident (see [3, Theorem 2] (Now86)).

For the remainder of this note we assume that $R$ is the ring $k\left[x_{1}, \ldots, x_{n}\right]$ of polynomials over $k$ and $\left\{\partial_{x_{1}}, \ldots, \partial_{x_{n-1}}, d\right\}$ is as in the previous theorem and also we recall the following definitions.

We recall from [7] that a $k$-derivation $d$ of $k\left[x_{1}, \ldots, x_{n}\right]$ is called locally nilpotent if for each $f \in R$ exists a natural number $n$ such that $d^{n}(f)=0$ and we say that a basis $\left\{d_{1}, \ldots, d_{n}\right\}$ of $\operatorname{Der}_{k}\left(k\left[x_{1}, \ldots, x_{n}\right]\right)$ is locally nilpotent if every derivation $d_{i}$ is locally nilpotent for $i=1, \ldots, n$.

We remember also that the Jacobian conjecture states that if $F=\left(F_{1}, \ldots, F_{n}\right)$ is a polynomial map such that the Jacobian matrix is invertible, then $F$ has a polynomial inverse (see [7]).

Theorem 7 (see [3, Theorem 5]). Let $R=k\left[x_{1}, \ldots, x_{n}\right]$ be the polynomial ring in $n$ variables over $k$. The following conditions are equivalent.

(1) The Jacobian conjecture is true in the n-variable case.

(2) Every commutative basis of the $R$-module $\operatorname{Der}_{k}(R)$ is locally nilpotent.

(3) Every commutative basis of the $R$-module $\operatorname{Der}_{k}(R)$ is locally finite.

Corollary 8. Let $\mathfrak{D}$ be a set of commute $k$-derivations of $R$ such that $R$ is $\mathfrak{D}$-simple, $\partial_{x_{1}}, \ldots, \partial_{x_{n-1}} \in \mathfrak{D}$, and the Jacobian 
conjecture is true in $R$. Then, there exists $d \in \mathfrak{D}$ such that $\left\{\partial_{x_{1}}, \ldots, \partial_{x_{n-1}}, d\right\}$ is a locally nilpotent commutative base of the $R$-module Der ${ }_{k}(R)$. In particular, $d$ is a $k$-derivation locally nilpotent.

Proof. The proof is immediate consequence of [3, Theorem 5].

Question. A ring is called $w$-differentially simple if it is a simple relative to a family with $w$ derivations. Recall that we are assuming $R=k\left[x_{1}, \ldots, x_{n}\right]$; then we know that $R$ is 1 differentially simple and $\operatorname{dim}(R)$-differentially simple as well. However, $n=\operatorname{dim}(R)$ is not necessarily the smallest $w$ for which such a ring can be $w$-differentially simple (see [8]). Thus, one may ask the following: what is the smallest positive integer $w \neq 1$ such that $R$ is $w$-differentially simple and all $w$ derivations are nonsimple and commute?

\section{Acknowledgment}

The research of Rene Baltazar was partially supported by CAPES of Brazil.

\section{References}

[1] J. Li and X. Du, "Pairwise commuting derivations of polynomial rings," Linear Algebra and Its Applications, vol. 436, no. 7, pp. 2375-2379, 2012.

[2] S. Maubach, "The commuting derivations conjecture," Journal of Pure and Applied Algebra, vol. 179, no. 1-2, pp. 159-168, 2003.

[3] A. Nowicki, "Commutative bases of derivations in polynomial and power series rings," Journal of Pure and Applied Algebra, vol. 40, no. 3, pp. 275-279, 1986.

[4] A. P. Petravchuk, "On pairs of commuting derivations of the polynomial ring in one or two variables," Linear Algebra and Its Applications, vol. 433, no. 3, pp. 574-579, 2010.

[5] K. Retert, "Sets of commuting derivations and simplicity," Communications in Algebra, vol. 34, no. 8, pp. 2941-2963, 2006.

[6] A. van den Essen, Polynomial Automorphisms and the Jacobian Conjecture, vol. 190 of Progress in Mathematics, Birkhäuser, Basel, Switzerland, 2000.

[7] A. Nowicki, Polynomial Derivations and Their Rings of Constants, N. Copernicus University Press, Torun, Poland, 1994.

[8] D. Levcovitz and S. C. Coutinho, "On the differential simplicity of affine rings," Proceedings of the American Mathematical Society. In press. 


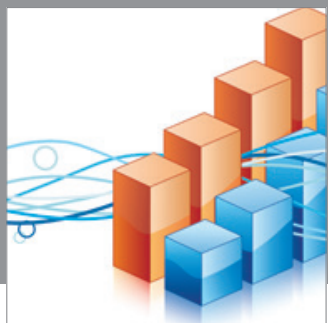

Advances in

Operations Research

mansans

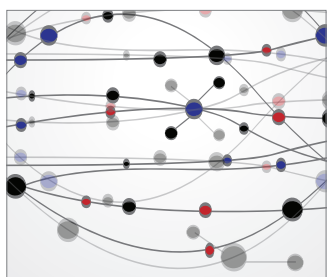

The Scientific World Journal
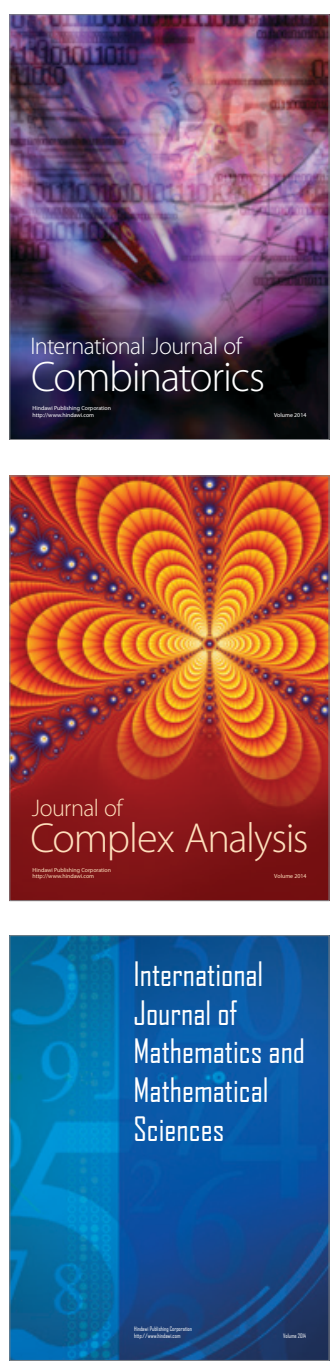
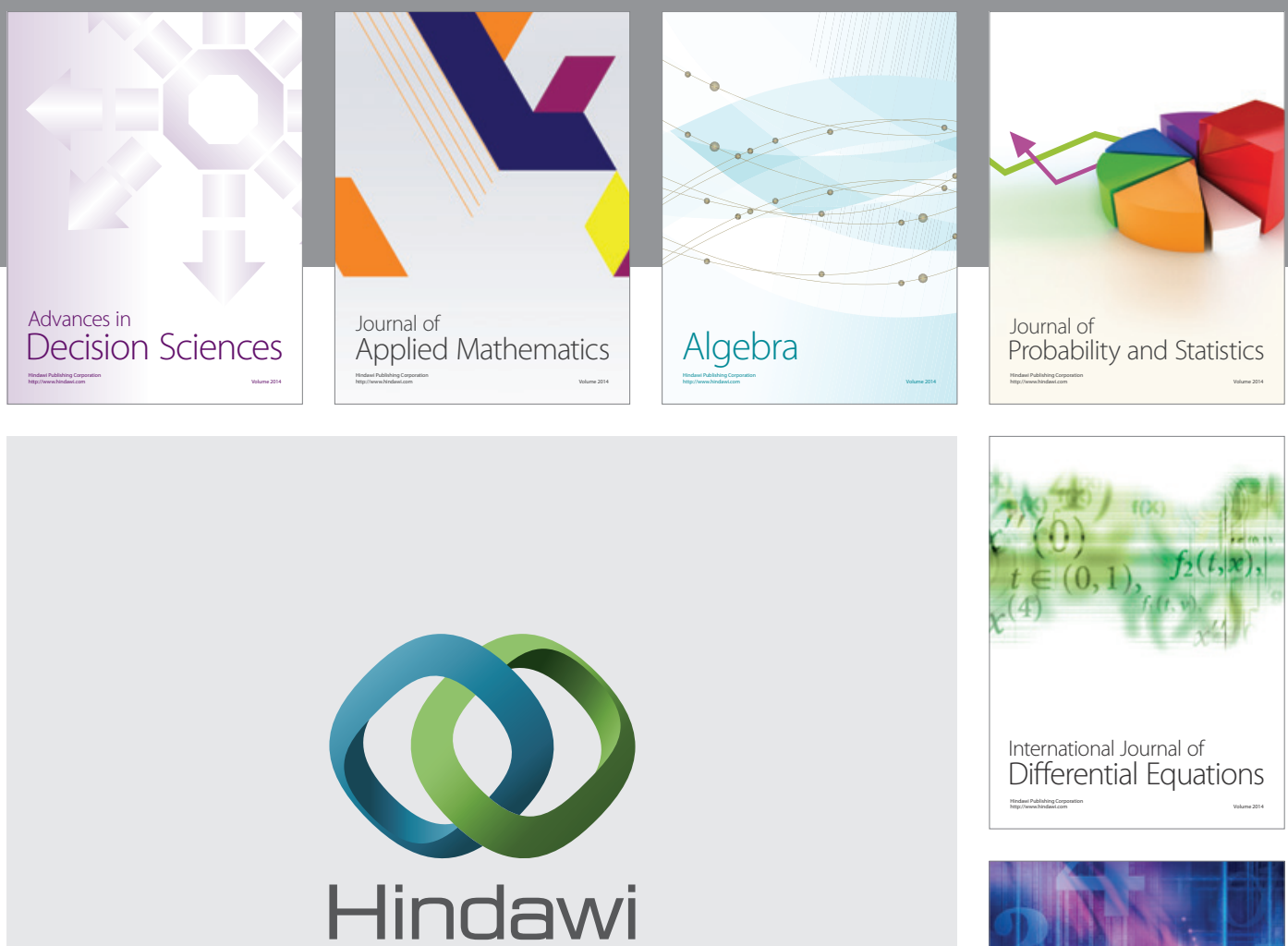

Submit your manuscripts at http://www.hindawi.com
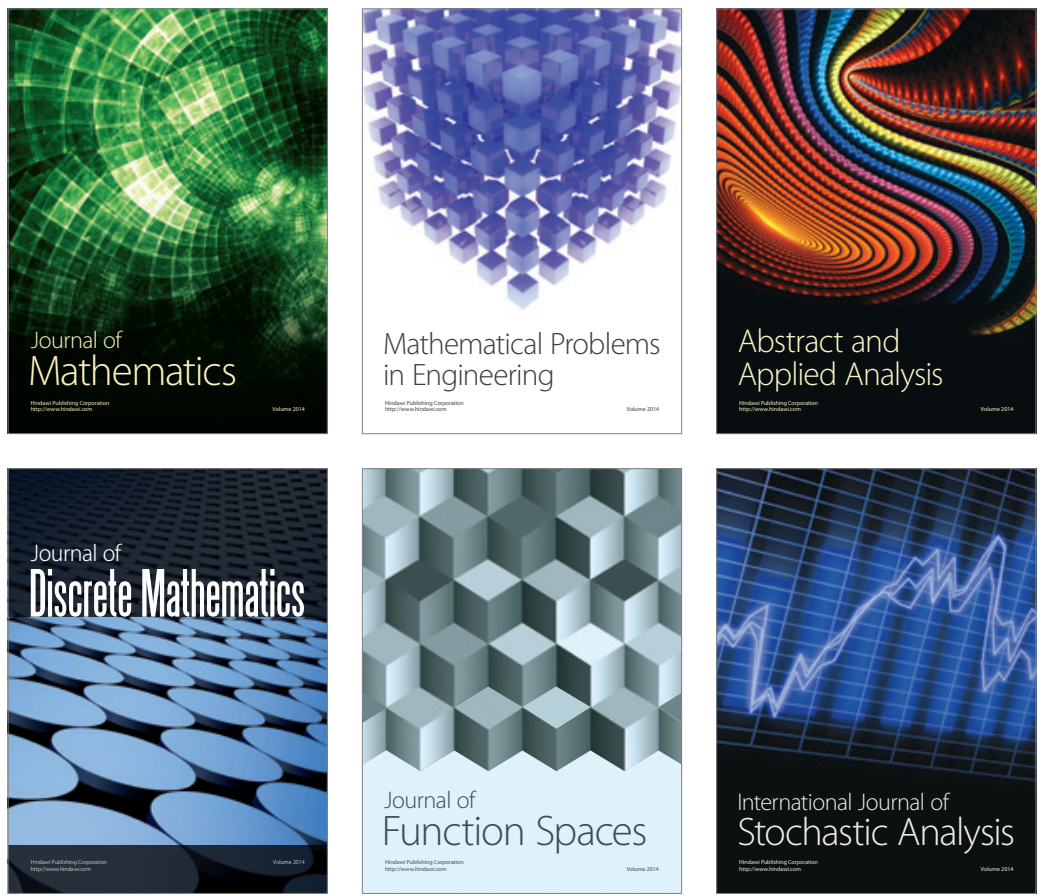

Journal of

Function Spaces

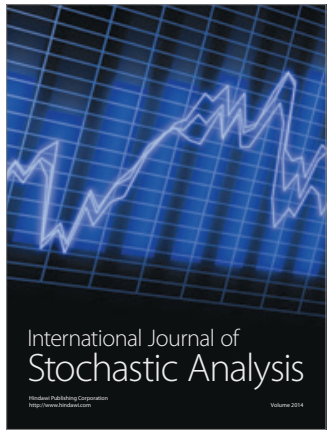

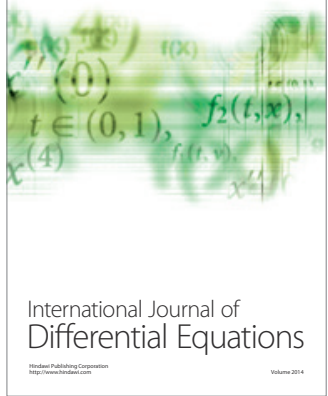
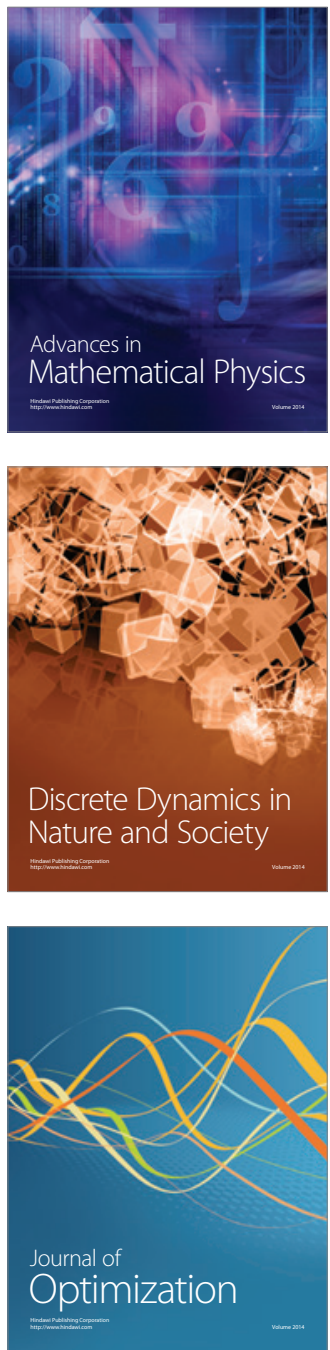